\title{
EIN3 binding site architecture guides transcriptional response to ethylene in Arabidopsis
}

\author{
Vladislav Dolgikh \\ ICG SB RAS, Novosibirsk, Russia \\ volleydollmc1@gmail.com
}

\author{
Victor Levitsky \\ ICG SB RAS, Novosibirsk, Russia \\ levitsky@bionet.nsc.ru \\ Elena Zemlyanskaya \\ ICG SB RAS, Novosibirsk, Russia \\ NSU, Novosibirsk, Russia \\ ezemlyanskaya@bionet.nsc.ru
}

\author{
Dmitry Oshchepkov \\ ICG SB RAS, Novosibirsk, Russia \\ diman@bionet.nsc.ru
}

\begin{abstract}
EIN3 transcription factor is the master regulator of gene expression in response to plant hormone ethylene. The structure of EIN3 binding site (EBS) was determined using in vitro binding assays. However, the role of EBS architecture in EIN3 binding at the whole-genome level is poorly understood. Here we demonstrate that an inverted repeat of EBS with the overlap of the motifs is a canonical EIN3 target in the Arabidopsis genome. We show that this EBS architecture provides a more pronounced response to ethylene than either a single EBS or EBS repeats with a spacer. These findings can be used to develop a new genetic sensor for highly sensitive detection of ethylene signaling in Arabidopsis.
\end{abstract}

Keywords - bioinformatics, transcription factor, ETHYLENE-INSENSITIVE3, EIN3 binding site (EBS), ChIPseq, RNA-seq

Motivation and aim

\section{Motivation}

ETHYLENE INSENSITIVE 3 (EIN3) transcription factor is the master regulator of gene expression in response to plant hormone ethylene that guides plant growth under stress conditions. EIN3 is a transcriptional activator that binds a short nucleotide sequence referred to as EBS to induce transcription [1]. Arabidopsis thaliana reporter EBS:GUS driven by such EIN3 binding site [2] is widely used as a sensor for detection of ethylene signaling. EIN3 tends to bind DNA as a homodimer and it has been shown recently that EBS inverted repeats with a spacer of $10 \mathrm{bp}$ provide EIN3 binding in vitro with a higher affinity than a single EBS [3]. However, the role of EBS architecture in EIN3 functioning was not investigated on the whole genome level.

Aim

Here we accomplish a systematic bioinformatics analysis of EIN3 bound sequences in Arabidopsis genome to shed light on molecular mechanisms utilized for regulation of transcriptional response to ethylene in plants.

\section{Methods}

We used publicly available ChIP-seq data on EIN3 binding, RNA-seq data on ethylene-induced transcriptomes in Arabidopsis seedlings [4] and published DAP-seq data [5]. We used Homer [6] for de novo motif search in the peaks, and MCOT [7] for enrichment analysis of EBS repeats. Associations of EBS configurations with peaks and genes features were estimated with Fisher's exact test.

\section{Results}

We discovered a previously unknown EBS architecture that is enriched in EIN3 bound sequences to a much greater extent than a single EBS motif. This new configuration is a head-to-head inverted repeat of EBS-like sequences with $1 \mathrm{bp}$ overlap referred to as $2 \mathrm{EBS}(-1)$. It is noteworthy that none of the EBS repeats with spaced motifs (including the one with 10 bp spacer) was enriched in EIN3 bound sequences compared to a random expectation. We also demonstrated that unlike a single EBS motif, 2EBS(-1) repeat was significantly associated with a sustained profile of EIN3 binding, i.e. it facilitated EIN3 binding regardless of the duration of ethylene treatment. Based on these findings we consider that 2EBS(-1) is a canonical EIN3 binding site in A. thailana genome. We further showed that of all EBS configurations under study only 2EBS(-1) was significantly associated with transcriptional response of EIN3 targets to ethylene treatment. Moreover, it tended to cause a more pronounced transcriptional response than other EBS configurations. These findings can be used to design a new genetic sensor for highly sensitive detection of ethylene signaling. Taken together, this work provides new insight on the molecular mechanisms utilized for regulation of transcriptional response to ethylene in plants.

\section{Acknowledgment}

Supported by the RSF (20-14-00140) and State Budget Project (0324-2019-0040).

\section{References}

[1] Kosugi S. and Ohashi Y. (2000) Cloning and DNA-binding properties of a tobacco Ethylene-Insensitive3 (EIN3) homolog. Nucleic Acids Res. 28(4):960-967.

[2] Stepanova A.N. et al. (2007) Multilevel interactions between ethylene and auxin in Arabidopsis roots. Plant Cell. 19(17):2169-2185.

[3] Song J. et al. (2015) Biochemical and Structural Insights into the Mechanism of DNA Recognition by Arabidopsis ETHYLENE INSENSITIVE3. PLoS One. 10(9):e0137439.

[4] Chang K.N. et al. (2013) Temporal transcriptional response to ethylene gas drives growth hormone cross-regulation in Arabidopsis. Elife. 2:e00675.

[5] O'Malley R.C. et al. (2016) Cistrome and Epicistrome Features Shape the Regulatory DNA Landscape. Cell. 165(5):1280-1292.

[6] Heinz S. et al. (2010) Simple combinations of lineage-determining transcription factors prime cis-regulatory elements required for macrophage and b cell identities. Mol. Cell. 38(4):576-589.

[7] Levitsky V. et al. (2019) A single ChIP-seq dataset is sufficient for comprehensive analysis of motifs co-occurrence with MCOT package. Nucleic Acids Res. 47(21):e139. 